\title{
Posición serial, densidad informativa y velocidad de lectura en el recuerdo de las cuñas de radio
}

\author{
Emma RODERO ${ }^{1}$ \\ Universitat Pompeu Fabra
}

\begin{abstract}
RESUMEN:
Uno de los objetivos prioritarios de la comunicación publicitaria es facilitar que el producto o servicio se recuerde después de la emisión en un intento de promover el consumo. El recuerdo de un anuncio radiofónico depende de varios factores que afectan a sus características estructurales. A nivel macroestructural, puesto que la publicidad radiofónica mayoritariamente adopta el formato de cuña, insertada en bloques publicitarios, resulta entonces importante determinar si el grado de recuerdo del contenido queda influido por la posición de la cuña dentro del bloque publicitario. Pero, junto a ello, existen otros elementos microestructurales que pueden influir en el grado de recuerdo de una cuña, como pueden ser la densidad informativa que contiene o la velocidad de lectura a la que se emite. Por tanto, esta investigación tiene como objetivo analizar si existe un efecto de posición serial en el recuerdo de las cuñas radiofónicas y si, unido a él, influyen otras peculiaridades del anuncio. Los resultados del estudio apuntan a que no es tan importante la posición en que se inserta la cuña radiofónica en el bloque publicitario como sus características microestructurales. Cuando un anuncio reúne escasa densidad informativa y elementos formales eficaces, además de incrementar el nivel de recuerdo, favorece que se produzca un efecto de posición serial. En cambio, éste no se produce cuando sus condiciones microestructurales no son favorables.
\end{abstract}

PALABRAS CLAVE: Cuñas radiofónicas; recuerdo; posición serial; densidad informativa; velocidad de lectura.

TITLE: Serial position, information density and speech rate in the recall of radio ads

\begin{abstract}
One of the main goals of commercial communication is the recall of the product or service in an attempt to promote the purchase. The recall of a radio ad depends on several factors affecting its structural characteristics. At the macro level, because most of the radio commercials take the format of an ad, inserted into commercial breaks, it is important to determine if the degree of recall of the content is influenced by the position of the ad into the advertising break. But, furthermore, the degree of recall of an ad can be influenced by other microstructural elements, such as its density of information or its speech rate. Therefore, this study is to analyze if a serial position effect is found in the recall of radio commercials and if this can be influenced by other characteristics of the ad. The findings of the study suggest that it is not so important the position of the ad in the radio advertising break as their microstructural characteristics. When an advertisement presents low information density and effective formal elements, as well as improving the recall level, promotes the emergence of a serial position effect. Instead, this phenomenon does not occur when the microstructural conditions are not favorable.
\end{abstract}

KEY WORDS: Radio ads; recall; serial position; information density; speech rate.

1 Emma Rodero. Facultad de Comunicación. Universitat Pompeu Fabra. emma.rodero@upf.edu 


\section{El recuerdo de la publicidad radiofónica}

La publicidad radiofónica en España lleva años sumida en una crisis expresiva que se manifiesta en un bajo nivel de calidad y, con ello, de efectividad (Rodero Antón, 2008; Muela Molina, 2008 y 2007; Balsebre et al., 2006; Marcos, 2006; Pérez, 2006; Barbeito Veloso y Fajula Payet, 2005; García, 1999). «Hoy por hoy la publicidad radiofónica destaca por su pobreza creativa y por un escasísimo repertorio de recursos expresivos, lo que da lugar a un paupérrimo lenguaje publicitario» ${ }^{2}$.

A pesar de ello, continúan siendo escasos los estudios que buscan determinar los efectos que estos mensajes producen en el oyente o potencial consumidor. Y todo ello cuando uno de los objetivos prioritarios de la comunicación publicitaria es precisamente facilitar que el producto o servicio se recuerde después de la emisión, precisamente para convertir al oyente en consumidor. Por tanto, esta tarea implica que el oyente codifique y almacene correctamente los datos del mensaje para ser capaz después de recuperar la información de la memoria. «Se puede decir que una publicidad que no ha sido codificada o que no puede ser recuperada, es como si no hubiera existido» ${ }^{3}$. Esta es la razón por la cual analizar el procesamiento cognitivo de la memoria de un oyente expuesto a un mensaje adquiere una importancia determinante dentro de la comunicación publicitaria.

El recuerdo de un anuncio radiofónico, como condición esencial de su efectividad, depende de varios factores que afectan a su macro y microestructura ( $\mathrm{Li}$, 2010). A nivel macroestructural, la publicidad radiofónica mayoritariamente adopta el formato de cuña insertada en bloques publicitarios. Por tanto, las cuñas no sólo compiten por la atención del oyente, sino también lo hacen con el resto de anuncios que se emiten en el mismo corte publicitario. Desde este punto de vista, resulta entonces importante determinar si el grado de recuerdo del contenido queda influido por la posición de la cuña dentro de estos bloques publicitarios. Esto es lo que se conoce como efecto de posición serial. A nivel microestructural, varios son los factores que pueden incidir sobre el recuerdo de las cuñas, desde la densidad de información que contienen hasta condicionantes formales como la velocidad de lectura. Partiendo de estas consideraciones, parece entonces pertinente realizar un estudio que analice el efecto de posición serial a nivel macroestructural y la posible influencia de otros factores microestructurales sobre el grado de recuerdo de un oyente expuesto a un bloque publicitario con varias cuñas radiofónicas.

2 GARCíA LÓPEZ, M. (2000): «Creatividad radiofónica, ¿para qué?», Questiones Publicitarias, 8, 60.

3 SÁIZ, M.; BAQUÉs, J. y SÁIZ, D. (1999): «Factores que pueden mejorar la codificación de los mensajes publicitarios: ¿una cuestión de memoria implícita o de memoria explícita?», Psicothema, 11(4), 892. 


\section{Factores macroestructurales que inciden sobre el recuerdo}

Dado que en la radio la publicidad se presenta al oyente en bloques publicitarios que contienen varias cuñas, la posición serial que ocupa cada mensaje es un factor importante de cara a su recuerdo. A pesar de ello, pocas investigaciones se han dedicado a analizar sus efectos (Brunel y Nelson, 2003; Broach et al., 1997).

El Efecto de Posición Serial (Position Serial Effect -PSE-) establece que el recuerdo de un estímulo contenido en una serie queda afectado por la posición en que se presente, puesto que su procesamiento implica dos tipos de almacenamiento en la memoria: la Memoria a Corto Plazo (MCP), de capacidad limitada, y la Memoria a Largo Plazo (MLP). Los numerosos trabajos realizados en el campo de la memoria han demostrado que se aumentan las posibilidades del recuerdo cuando los estímulos presentados en una serie se encuentran al inicio, lo que se conoce como efecto de primacía, y al final, el denominado efecto de recencia (Oberauer, 2003). Por tanto, cuando se realiza un gráfico de recuerdo de posición serial se obtienen curvas típicas con un nivel alto al principio y al final y una notable caída en los elementos intermedios (Glanzer y Cunitz, 1966), como puede observarse en el gráfico 1.

Fig. 1. Curva de posición serial

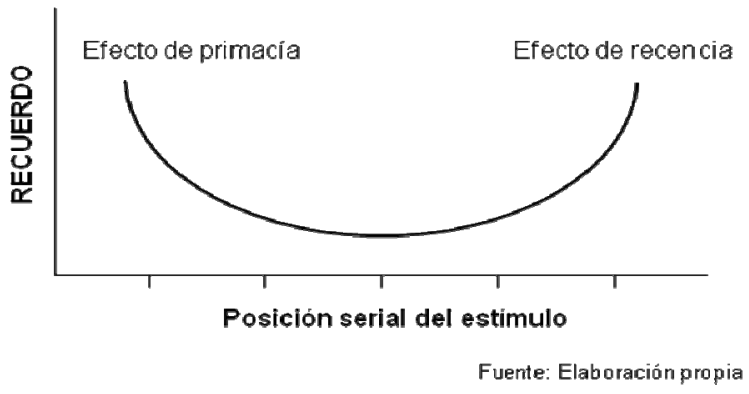

La explicación de este fenómeno se basa en que la primera información de una serie recibe teóricamente mayores índices de atención porque el oyente se siente más persuadido (Brunel y Nelson, 2003), con lo que la información se transfiere a la MLP, y puede ser recuperada posteriormente (Rundus, 1971). En consecuencia, se considera que el efecto de primacía se relaciona con procesos controlados de nivel superior. En cambio, los elementos intermedios, con menor posibilidad de repetición, permanecen en la MCP, de la cual son sustituidos por las últimas unidades de la serie. Por eso, presentan menor nivel de recuerdo. Los elementos finales de la serie obtienen mejores índices porque al final de la exposición aún permanecen en la MCP, gracias a su cercanía o proximidad temporal, y no son interferidos por un estímulo posterior. De esta manera, el efecto de recencia se sustenta en la $\mathrm{MCP}$, es decir, en un proceso cognitivo de nivel inferior. 
Uno de los pocos estudios aplicado a la publicidad radiofónica confirma que se recuerdan más aquellos bloques de anuncios que van en primera y en última posición con un efecto más acentuado para el efecto de primacía (Riebe y Dawes, 2006). Otro estudio aplicado a la radio, si bien a las noticias (Tannenbaum, 1954), ratifica los efectos de primacía y recencia pero con índices más elevados en recencia. El problema de este estudio es que no varía el orden interno de los estímulos. El resto de los estudios que han comprobado el efecto de posición serial se centran en el análisis de la publicidad televisiva. Los resultados del estudio de Zhao (1997) sobre el recuerdo de las marcas concluyen que un anuncio dentro de un bloque publicitario con pocos spots, colocado en una posición inicial, refuerza el recuerdo de la marca. Estos mismos resultados se repitieron en la investigación de Pieters y Bijmolt (1997). El estudio de Brunel y Nelson (2003) revela, además de un efecto de posición serial, diferencias de preferencia por género. Las mujeres recordaron mejor el mensaje presentado en primera posición mientras que en los hombres fue el último. Estos resultados confirman que en situaciones con baja implicación, como puede ser el caso de la exposición a un anuncio radiofónico, cuando no se evalúa el contenido, el orden de presentación presenta diferencias de género. Junto a ello, la investigación realizada por Saiz et al. (1999), aplicada a los spots televisivos, demuestra que se observa muy claramente un efecto de de posición serial que hace que los primeros y especialmente los últimos anuncios sean los más recordados. Como puede observarse, por tanto, más allá de comprobar el efecto de posición serial, cada estudio obtiene diferentes resultados sobre cuál es el principio dominante, si primacía o recencia. El nivel de recuerdo puede ser en unos casos más alto al inicio de la serie, potenciando el efecto de primacía, o bien al final de la misma, reforzando el efecto de recencia (Murdock, 1962), pero siempre depende de los condicionantes del estímulo presentado. En concreto, cuando la modalidad es auditiva, como ocurre en la radio, los estudios indican que debería potenciarse en mayor medida el efecto de recencia.

Existen dos conjuntos de factores que a nivel macroestructural pueden influir en que los elementos de una serie puedan ser mejor o peor recordados según su posición. El primero de ellos tiene que ver con el grado de implicación del sujeto en la exposición al estímulo como consecuencia de su interés y actitud hacia el mensaje (Brunel y Nelson, 2003). Algunas investigaciones previas demuestran que cuando un sujeto tiene un elevado interés en un estímulo se potencia el efecto de primacía mientras que cuando el interés es bajo se refuerza el efecto de recencia (Lana, 1963). También el efecto de recencia se potencia cuando los sujetos no son motivados para procesar la información $\mathrm{y}$, por tanto, no prestan excesiva atención al estímulo (Haugtvedt y Wegener, 1994). El problema es que, aún siendo un factor que ha de tenerse presente, lo cierto es que resulta de difícil control por parte de los publicistas.

Existe otro conjunto de factores que a nivel macroestructural tiene relación con el estímulo. Algunas investigaciones han demostrado que el recuerdo se favorece cuando el oyente puede establecer cierto grado de asociación entre los estímulos (Ballesteros, 2001; Ballesteros y García, 1998). Pero este es un factor que no se 
produce en las series de cuñas insertadas en un bloque publicitario por su condición de contenido autónomo e independiente. En cambio, el otro elemento determinante que puede afectar al recuerdo sí presenta una clara aplicación. Algunos autores han demostrado que la frecuencia de repetición del estímulo influye sobre la memoria (Jurafsky 1996; Corley y Crocker, 2000). Puesto que las cuñas radiofónicas se presentan en varias emisiones, éste sería un factor que podría aumentar su nivel de recuerdo, si bien no es objeto de estudio en esta investigación.

En cambio, uno de los elementos que puede conllevar efectos negativos sobre el recuerdo de la publicidad en la radio es la condición de escucha del oyente. Normalmente la audiencia no se encuentra en una disposición inicial ni de prestar atención ni de esforzarse por almacenar datos en la memoria, más bien la situación de la recepción es adversa sin un acto volitivo o intencional de memoria (Sáiz et al., 1999), a lo que se añade el hecho de que la mayoría de los oyentes escuchan la radio realizando una actividad paralela. No olvidemos que los datos indican que el $80 \%$ de los oyentes están haciendo otra cosa al mismo tiempo (Joannis, 1996). Algunos autores (Terry, 2005; Atkinson y Shiffrin, 1968; Postman y Phillips, 1965) han demostrado que una tarea distractora puede afectar a los efectos de primacía y recencia, por lo que, al menos teóricamente, los oyentes se encuentran en una posición poco favorable al recuerdo. En estas condiciones normalmente prima el efecto de recencia.

En definitiva, estas consideraciones nos permiten formular las dos primeras hipótesis de este estudio:

Hipótesis 1: Las cuñas radiofónicas emitidas en primera y última posición de un bloque publicitario obtendrán un mejor nivel de recuerdo que aquellas que se encuentran en las posiciones intermedias.

Hipótesis 2: Los bloques publicitarios expuestos con modalidad de escucha aislada obtendrán mejor grado de recuerdo que aquellos expuestos con modalidad de escucha compartida, mientras el oyente realiza una actividad paralela.

Junto a estos factores que afectan a la macroestructura, el recuerdo de una cuña radiofónica puede verse también influido a nivel microestructural por las propias características del mensaje que sí dependen directamente del trabajo del publicista.

\section{Factores microestructurales que inciden sobre el recuerdo}

De acuerdo con el modelo de procesamiento de capacidad limitada (Limited Capacity Model of Motivated Mediated Message Processing -LC4MP-, Lang, 2009, 2006 y 2000), base de este estudio, el número de recursos que requiere un sujeto para procesar un mensaje depende de dos condicionantes del estímulo: su densidad informativa y su complejidad estructural y formal. Puesto que para procesar un mensaje, como puede ser el radiofónico, un sujeto dispone de unos recursos que son limitados, cuando se incrementa su complejidad, el procesamiento puede 
no completarse correctamente debido a una sobrecarga cognitiva -cognitive overload- (Fox et al., 2007).

En primer lugar, la densidad informativa, es decir, el volumen global de datos que contenga un mensaje puede ser un elemento determinante en su grado de recuerdo. Cuantos más datos presente un estímulo, más difícil será su procesamiento auditivo $\mathrm{y}$, con ello, el riesgo de sobrecarga cognitiva será más elevado. Esto es lo que han comprobado Potter et al. (2006) en un estudio aplicado a la radio. Cuando se incrementaba la densidad de información, se producía una sobrecarga cognitiva que afectaba al recuerdo. En consecuencia, debido a la limitada capacidad de procesamiento en la radio, es importante que los mensajes no contengan una elevada carga informativa (Rodero Antón et al., 2004). A pesar de estos datos, no es difícil comprobar cómo la mayoría de las cuñas radiofónicas emitidas en las emisoras españolas presentan una elevada densidad de datos referidos al producto o servicio que se anuncia.

En segundo lugar, las características estructurales de la cuña radiofónica constituyen otro factor que puede influir sobre el grado de recuerdo del contenido. Desde este punto de vista, la estructura narrativa que adopta puede favorecer o perjudicar el nivel de memoria del oyente. Básicamente se pueden diferenciar dos tipos de estructuras: la informativa y la dramatizada. Varios estudios han demostrado que la estructura dramatizada favorece la atención y facilita el recuerdo, gracias a la percepción de realismo (Press, 1989) y a la identificación del oyente con los personajes (Cohen, 2001). Sin embargo, las investigaciones realizadas sobre la publicidad radiofónica indican que la tendencia es la contraria. El estudio de Perona Páez (2007) indica que la mayoría de las cuñas radiofónicas presentan una estructura narrativa claramente informativa y el de Muela Molina (2008) revela una considerable representatividad de anuncios con características del producto o datos del servicio.

Por último, las características formales del mensaje, a través de la utilización de los elementos del lenguaje radiofónico, también son factores que pueden incidir sobre el nivel de recuerdo.

En lo referente a la palabra, uno de los mayores condicionantes de una cuña radiofónica en relación con su capacidad de comprensión es la velocidad de lectura del locutor. Son varios los autores que han demostrado que afecta directamente al nivel de recuerdo de un mensaje (Murphey et al., 2003; Meyerson, 1974; Goldhaber, 1974). Los estudios de Atkinson y Shiffrin (1968) han comprobado que la tasa de presentación del estímulo afecta de manera negativa al principio de primacía, aunque no al de recencia. Resulta lógico pensar que cuando se incrementa la velocidad de lectura, el oyente dispone de menos tiempo para procesar la información con unos recursos que, además, son limitados, por lo que en último término resulta afectada la memoria. Más aún Jahnke (1968) ha evaluado los efectos de la tasa de presentación en el efecto de posición serial en recuerdo libre. Si bien se trata de estudios donde se presentan palabras aisladas, estos autores demuestran que cuando los estímulos se presentan a una tasa más lenta se mejora el recuerdo y viceversa. Aplicado a la radio, la mayoría de los autores coinciden en señalar que una óptima 
velocidad de lectura podría establecerse entre las 160 y 180 palabras por minuto (Chantler and Stewart, 2003; Boyd, 2003). Sin embargo, algunos estudios realizados, si bien en las noticias, elevan este umbral hasta las 200 palabras por minuto (Rodero Antón, 2007). Y, en este caso, la publicidad no es una excepción.

Es importante también, en cuanto al contenido, denunciar la sobreexplotación del texto publicitario en radio con respecto al tiempo de emisión. Nos encontramos habitualmente con demasiados datos en pocos segundos, leídos a una velocidad elevada (para que encajen en ese tiempo), con lo que la escucha y compresión de la publicidad se resiente ${ }^{4}$.

Otro aspecto relacionado con la voz que puede afectar directamente al recuerdo es la dicción o correcta articulación, si bien éste es un aspecto que suele estar muy cuidado en la publicidad nacional al contar con locutores profesionales. En cambio, el género del locutor no es un factor que afecte al procesamiento del mensaje. Un estudio realizado sobre este aspecto demuestra que no existen diferencias significativas cuando la voz es de un locutor frente a la de una locutora (Rodero et al., 2010). Pese a estos datos, los estudios realizados en España por Perona Páez y Barbeito Veloso (2008) han cuantificado una fuerte presencia de la voz masculina en la publicidad radiofónica española: un 73 por ciento frente a tan sólo un 27 por ciento de voces femeninas.

Por último, tanto la utilización de la música como de los efectos sonoros en una cuña radiofónica pueden favorecer el recuerdo de los datos (Potter y Choi, 2006; Potter y Callison, 2000). Pero el empleo de estos recursos en la publicidad radiofónica no suele ser muy afortunado. Primero, es cierto que la mayoría de las cuñas radiofónicas utilizan música pero la emplean como adorno, con carácter puramente ornamental (Vázquez Gestal, 2001), por tanto, sin que exista una función precisa que pueda favorecer el recuerdo. Segundo, el carácter estructural informativo de la mayoría de las cuñas radiofónicas no favorece la utilización de efectos sonoros, por lo que la mayoría no hacen uso de este recurso.

De todos los factores aquí descritos existen dos que presentan una mayor variabilidad en la publicidad radiofónica española y que por influir de forma directa sobre el recuerdo de una cuña serán los que se establezcan como referencia para el estudio. El primero de ellos es la densidad informativa, dependiente del contenido del mensaje, con bastante variabilidad, y que muchos estudios coinciden en señalar como destacada por su incidencia directa en el grado de complejidad del procesamiento auditivo. El segundo de ellos de carácter formal es la velocidad de lectura que también presenta una influencia directa sobre el correcto procesamiento auditivo del mensaje. El resto de los factores o bien no son tan variables o no son tan determinantes en el grado de recuerdo, como ocurre con la estructura narrativa o lo elementos formales como el tipo de voz, la dicción (correcta en la publicidad pro-

4 Rodero Antón, E. (2008): «Publicidad en radio sí, pero no radiofónica», Área Abierta, 20, 11. 
fesional) o la utilización de música o efectos de sonido. Estos elementos favorecen la memoria gracias a que incrementan la atención y, con ello, una posibilidad mayor de recuerdo, por lo que su incidencia es indirecta. Así pues estas consideraciones nos conducen a formular la tercera hipótesis de este estudio:

Hipótesis 3: Las cuñas radiofónicas con menor densidad informativa y velocidad de lectura obtendrán un mejor nivel de recuerdo que aquellas con índices más elevados en ambas variables.

\section{Metodología}

El objeto de estudio de esta investigación son las cuñas de radio entendidas como «forma compacta de publicidad en radio que se caracteriza por el hecho de ser breve, repetible y sin relación con la programación en la cual se inserta» ${ }^{5}$ y que se emiten dentro de un bloque publicitario. Se ha escogido este formato al ser el más habitual en la publicidad radiofónica en España (Asociación Española de la Publicidad, 2011; Perona Páez y Barbeito Veloso, 2008; Perona Páez, 2007), por tanto, el que presenta mayor volumen de inversión por formatos (Infoadex, 2011).

\subsection{Preselección del corpus del estudio}

Puesto que la prueba de esta investigación se realiza con sujetos experimentales cuya lengua materna es el catalán, en una primera fase se procedió a recoger un corpus inicial de publicidad radiofónica en lengua catalana del magacín con mayor audiencia en Cataluña El matí de Catalunya Ràdio, (347.000 oyentes diarios), por encima del magacín con mayor audiencia en el resto del territorio español (Hoy por Hoy de la Cadena Ser), según los datos de la primera oleada del EGM en 2011. Se trataba de buscar un programa en catalán destinado a un público heterogéneo para evitar publicidad dirigida a segmentos específicos de audiencia. De esta manera, durante una semana, entre el lunes 24 y el viernes 28 de febrero de 2011, entre las 10,00 y las 12,00 , se registraron un total de 31 bloques publicitarios con un total de 72 cuñas, de las cuales 13 fueron eliminadas al ser repetidas. Por tanto, la recogida de la fase inicial quedó conformada por una muestra de 59 cuñas radiofónicas sobre las cuales realizar un análisis de los criterios microestructurales:

- Estructurales: duración y estructura narrativa (informativa o dramática).

- Formales: número de locutores, género de la voz del locutor, utilización o no de música, utilización o no de efectos sonoros.

5 PeRONA PÁEZ, J.J. (2007): «Formatos y estilos publicitarios en el prime-time radiofónico español: infrautilización y sequía de ideas», Zer, 23, 224. 
Los resultados de este análisis inicial indicaron, en línea con investigaciones previas, que la mayoría de las cuñas tenían una duración de 20 segundos $(22,5$ segundos de media), que la estructura narrativa mayoritaria era informativa $(71 \%)$, que la mayoría eran presentadas por un sólo locutor (62\%), generalmente de género masculino $(76 \%)$ con dicción correcta, y que normalmente no empleaban efectos de sonido (37\%) aunque sí música de manera ornamental (87\%). Estas características conformarían las constantes del estudio.

\subsection{Selección del corpus definitivo y determinación de variables independientes}

Para seleccionar el corpus final del estudio se escogieron, entonces, cuatro cuñas con estas características de empresas o productos nacionales para eliminar el efecto localista. Se trataba de evitar un alto nivel de variabilidad y, con ello, el efecto distintivo -distinctiveness effect- (Terry, 2005; Hunt, 1995), es decir, que alguno de los anuncios sobresaliera por encima del resto. Por tanto, estas cuatro cuñas conformarían el corte publicitario a analizar. Es evidente que los bloques de otras cadenas radiofónicas contienen un número más elevado de cuñas, una media de siete según el estudio de Muela Molina (2001), si bien algunos sobrepasan la veintena. Pero, como la misma autora considera:

Hay que tener mucha fe para pensar que un mensaje emitido antes y después de otros datos tan similares, sin destacar ninguno sobre los restantes, todos muy informativos, con una exagerada cantidad de datos, y desconectados del contexto programático donde son incluidos, puedan ejercer alguna influencia sobre el receptor ${ }^{6}$.

Además, el estudio de Potter (2009) demuestra que el recuerdo se incrementa cuando los bloques de publicidad contienen menos anuncios que cuando las unidades son más elevadas. Este autor corrobora los estudios de Tse et al. (2004) que establecen un mayor esfuerzo cognitivo, que puede desconectar al oyente, alrededor de los 110 segundos, es decir, en torno a las cuatro cuñas de unos 25 segundos. Por su parte, Webb y Ray (1979) mostraron un efecto de posición serial en grupos de cuatro anuncios. Además, los bloques más extensos registrados en la emisora de análisis estaban formados por cuatro cuñas radiofónicas, por lo que ésta sería la cantidad que se tomaría como referencia a la hora de conformar la serie.

Los anuncios seleccionados en esta fase previa variaban en los dos aspectos microestructurales de incidencia directa sobre el recuerdo que se buscaba investigar: la densidad informativa y la velocidad de lectura del locutor, que quedaban así incluidas en la variable independiente cuña publicitaria. La densidad informativa se midió estableciendo el porcentaje entre las palabras fundamentales en el contenido de la cuña, es decir, los contenidos referidos al mensaje comercial que conforma-

6 Muela Molina, C. (2001): La publicidad radiofónica en España. Análisis creativo de sus mensajes, Madrid: Ediciones Internacionales Universitarias, 243. 
ban un argumento (datos esenciales, marca y producto) en relación con el número total de palabras del anuncio, es decir, con los datos secundarios (Rodero Antón et al., 2004). La velocidad de lectura se midió en palabras por minuto. Finalmente el corpus definitivo para el estudio experimental quedó conformado por cuatro cuñas que presentaban las siguientes características:

Tabla 1. Características del estímulo

\begin{tabular}{|c|c|c|c|}
\hline Cuñas & Constantes & $\begin{array}{l}\text { Velocidad } \\
\text { de lectura }\end{array}$ & $\begin{array}{l}\text { Densidad } \\
\text { informativa }\end{array}$ \\
\hline $\begin{array}{l}\text { 1. El Corte Inglés (Alimenta- } \\
\text { ción) }\end{array}$ & - Un locutor, voz masculina & 171,4 & $9 \%$ \\
\hline 2. Campeonatos de Trial y & $\begin{array}{l}\text { - } 20 \text { segundos de duración } \\
\text { - Estructura informativa }\end{array}$ & 212,6 & $26,5 \%$ \\
\hline 3. Fundación Repsol (Energía) & $\begin{array}{l}\text { - Música ornamental origi- } \\
\text { nal en toda la cuña }\end{array}$ & 238,9 & $30,2 \%$ \\
\hline $\begin{array}{l}\text { 4. La Caixa (Servicios banca- } \\
\text { rios) }\end{array}$ & - Sin efectos de sonido & 185,4 & $19,6 \%$ \\
\hline
\end{tabular}

Fuente: Elaboración propia.

De esta manera, las cuñas con una densidad informativa inferior al 20 por ciento $\mathrm{y}$, con ello, compuestas por uno o dos argumentos a lo sumo, como recomienda Muela Molina (2001), fueron interpretadas como baja densidad (El Corte Inglés y La Caixa) y, a partir de este límite, con más de tres argumentos, como alta densidad. Asimismo aquellas con una velocidad de lectura establecida como óptima se clasificaron como de velocidad moderada frente a las que superaban las 200 palabras por minuto con velocidad alta (Fundación Repsol y Campeonatos). Con estas cuñas se formaron cuatro bloques publicitarios combinando la posición serial y estableciendo así otra variable independiente:

$\begin{array}{llll}\text { Bloque 1: Cuña } 1 & \text { Cuña } 2 & \text { Cuña } 3 & \text { Cuña } 4 \\ \text { Bloque 2: Cuña } 2 & \text { Cuña } 1 & \text { Cuña } 4 & \text { Cuña } 3 \\ \text { Bloque 3: Cuña } 3 & \text { Cuña } 4 & \text { Cuña } 2 & \text { Cuña } 1 \\ \text { Bloque 4: Cuña } 4 & \text { Cuña } 3 & \text { Cuña } 1 & \text { Cuña } 2\end{array}$

Para simular una emisión real, los cuatro bloques llevaban una ráfaga de separación al inicio y al final. Esta ráfaga era real y correspondía a la que el mismo programa analizado de Catalunya Ràdio emplea como separador de la publicidad. La duración total de cada bloque fue de un minuto y 40 segundos.

Pero como los oyentes suelen realizar una actividad al mismo tiempo que escuchan la radio, en esta investigación los sujetos se expusieron al mensaje en dos modalidades de escucha diferentes: la escucha aislada y la escucha compartida. Dos de estos grupos ( 2 y 4 ) escucharon al estímulo mientras jugaban al Buscaminas, un sencillo juego de Windows, que no requiere un alto grado de concentración. 
Los otros dos grupos, correspondientes a los bloques 1 y 3 de publicidad, se expusieron al estímulo sin realizar otra actividad paralela. De esta manera, la condición de escucha formaría otra variable independiente. Por último, puesto que el estudio de Brunel y Nelson (2003) demuestra diferencias en el efecto de posición serial por género, la última variable independiente quedaba conformada por el sexo de los miembros de la muestra.

\subsection{Procedimiento experimental}

La muestra de sujetos experimentales quedó conformada por 80 estudiantes universitarios de publicidad $(\mathrm{N}=80)$, con una media de 22 años y un alto nivel de competencia en lengua catalana, testada a través de una pregunta previa al cuestionario del estudio. Si bien las características de esta muestra no la hacen público objetivo de la publicidad seleccionada para el estudio, lo cual puede reducir su motivación, éste hecho puede ser compensado por ser objeto de análisis de los estudios que realizan. El grupo total fue dividido aleatoriamente en subgrupos de 20 guardando la proporción de género. De esta manera, cada grupo escuchó sólo uno de los cuatro bloques publicitarios.

La exposición al estímulo se realizó en una sala con óptimas condiciones acústicas, donde fueron conducidos cada uno de estos grupos a horas diferentes. Una vez que los sujetos escucharon su correspondiente bloque publicitario, procedieron a rellenar el cuestionario. La duración total del experimento fue de 40 minutos.

\subsection{Recuerdo como variable dependiente}

La medición del recuerdo, como variable dependiente, se empleó para evaluar el subproceso cognitivo de almacenamiento de información en la memoria para su posterior recuperación (Lang, 2009). Por eso, resulta apropiada para este estudio si tenemos en cuenta que éste es efecto último que debe producir una cuña publicitaria en un oyente si pretende incitarle a la compra final del producto. En general, la mayoría de los estudios emplean como método de evaluación para este subproceso un cuestionario de recuerdo libre, compuesto por preguntas que, en ausencia del estímulo, el sujeto experimental debe responder sin ninguna clave que le indique lo que ha de recordar.

El cuestionario de recuerdo libre, disponible en el anexo, estaba conformado por seis preguntas abiertas, dos de ellas sobre la idea que destacaba la cuña o contenidos secundarios (por tanto, no se preguntaba por datos) y las cuatro restantes sobre datos esenciales del anuncio (contenidos referidos al mensaje comercial, entre ellos, el producto y la marca). Es importante destacar que, frente a la mayoría de experimentos en los que se orienta la atención de los sujetos a la tarea de recordar determinados datos del estímulo, los oyentes participantes en esta investigación no recibieron instrucciones sobre lo que se les pediría después. Este factor puede, sin 
duda, reducir los niveles de recuerdo y potenciar el efecto de recencia sobre el de primacía (Haugtvedt y Wegener, 1994), pero se entendía que esta es la condición de escucha en que un oyente se expone habitualmente a la publicidad radiofónica. Por último, en el cuestionario debían escribir en dos líneas una explicación sobre las razones que les habían llevado a recordar las cuñas en mayor o menor grado.

\section{Resultados}

Para comprobar las hipótesis de este estudio, se ha realizado un análisis factorial de medidas repetidas UNIANOVA compuesto por 4 (cuña publicitaria) por 4 (posición serial de la cuña) por 2 (condición de escucha) por 2 (sexo de los sujetos) aplicado a la variable dependiente recuerdo. Mediante un nivel alfa de .001 para evaluar la homogeneidad, el test de homogeneidad de varianza de Levene no fue significativo $(p=.012)$ lo que no hace posible asumir que se cumple el principio de homocedasticidad.

De todos los casos analizados, las diferencias fueron estadísticamente significativas tanto para la cuña publicitaria $(F=15,86, p<.000)$, como para la condición de escucha $(F=12,35, p<.000)$, así como para la interacción entre la cuña publicitaria y su posición serial $(F=4,44, p<.000)$. En cambio no fueron significativos para la posición serial de las cuñas $(F=3,44, p<.017)$ ni para el sexo de los sujetos experimentales $(F=1,20, p<.234)$. Estos resultados se observan en la tabla 2:

Así pues, puesto que la posición serial de las cuñas dentro del bloque publicitario no obtiene diferencias significativas, los resultados no permiten confirmar la primera hipótesis, según la cual las cuñas radiofónicas emitidas en primera y última posición de los diferentes bloques publicitarios obtendrían un mejor nivel de recuerdo que aquellas que se encontraban en las posiciones intermedias. Por sí misma, la posición dentro de la serie no obtiene significación si no es en la interacción con la cuña. Esto revela, por tanto, que las características de cada anuncio están interaccionando en la variable recuerdo.

Si analizamos los bloques publicitarios (la interacción entre la cuña y su posición serial), observamos que el que obtiene una media más elevada de recuerdo ha sido el primero, con una modalidad de escucha aislada, seguido del tercero (modalidad aislada), el cuarto (modalidad compartida) y, por último, el segundo (modalidad compartida). Esto demuestra con diferencias significativas que se han recordado mejor los bloques escuchados sin realizar una actividad paralela $(M=1,23, D T$ $=1,46)$ que aquellos en los que se sí se ejecutaba $(M=, 53, D T=, 92)$. Aplicando un test post-hoc, los resultados indican que las diferencias son significativas entre todos los bloques publicitarios. Por lo tanto, estos datos permiten confirmar la segunda hipótesis de este estudio. 
Tabla 2. Medias y desviaciones típicas de las cuñas por bloques publicitarios

\begin{tabular}{|c|c|c|c|c|}
\hline Posición serial & $\begin{array}{c}\text { Modalidad de } \\
\text { escucha }\end{array}$ & Cuña & Media & DT \\
\hline \multirow{5}{*}{$\begin{array}{c}\text { Bloque } 1 \\
\text { (El Corte Inglés-Campeonatos- } \\
\text { Fundación Repsol-La Caixa) }\end{array}$} & \multirow{5}{*}{$\begin{array}{c}\text { Sin actividad } \\
\text { paralela }\end{array}$} & Primero & 2,75 & 1,90 \\
\hline & & Segundo &, 70 & 1,61 \\
\hline & & Tercero & ,20 & ,61 \\
\hline & & Cuarto & 1,40 & 1,26 \\
\hline & & Total & 1,26 & 1,82 \\
\hline \multirow{5}{*}{$\begin{array}{c}\text { Bloque } 2 \\
\text { (Campeonatos-El Corte Inglés- } \\
\text { La Caixa-Fundación Repsol) }\end{array}$} & \multirow{5}{*}{$\begin{array}{c}\text { Con actividad } \\
\text { paralela }\end{array}$} & Primero &, 33 & ,97 \\
\hline & & Segundo &, 53 &, 76 \\
\hline & & Tercero &, 35 & ,45 \\
\hline & & Cuarto & 09 &, 32 \\
\hline & & Total & ,29 &, 68 \\
\hline \multirow{5}{*}{$\begin{array}{l}\text { Bloque } 3 \\
\text { (Fundación Repsol-La Caixa- } \\
\text { Campeonatos-El Corte Inglés) }\end{array}$} & \multirow{5}{*}{$\begin{array}{l}\text { Sin actividad } \\
\text { paralela }\end{array}$} & Primero &, 70 & 1,04 \\
\hline & & Segundo & 1,46 & 1,23 \\
\hline & & Tercero & 0,85 & 1,25 \\
\hline & & Cuarto & 1,09 & ,33 \\
\hline & & Total & 1,10 & 1,19 \\
\hline \multirow{5}{*}{$\begin{array}{c}\text { Bloque } 4 \\
\text { (La Caixa-Fundación Repsol-El } \\
\text { Corte Inglés-Campeonatos) }\end{array}$} & \multirow{5}{*}{$\begin{array}{c}\text { Con actividad } \\
\text { paralela }\end{array}$} & Primero & 1,24 & ,80 \\
\hline & & Segundo &, 29 &, 55 \\
\hline & & Tercero & 1,44 & 1,49 \\
\hline & & Cuarto &, 46 &, 72 \\
\hline & & Total &, 86 & 1,06 \\
\hline \multirow{5}{*}{ Total } & & Primero & 1,25 & 1,48 \\
\hline & & Segundo &, 75 & 1,18 \\
\hline & & Tercero &, 71 & 1,13 \\
\hline & & Cuarto &, 83 & 1,08 \\
\hline & & Total &, 89 & 1,30 \\
\hline
\end{tabular}

Fuente: elaboración propia.

Si analizamos las cuñas, aspecto que también ha sido significativo, el orden de recuerdo ha sido el siguiente: El Corte Inglés, La Caixa, Campeonatos y Fundación Repsol. Este orden coincide precisamente con una tasa creciente tanto en densidad de información como en velocidad de lectura. De hecho, si aislamos las cuñas con una menor densidad informativa y velocidad moderada $(M=1,21, D T=1,34)$ y las comparamos con aquellas que tienen mayor densidad y velocidad rápida $(M=$ ,48, $D T=1,01)$, los resultados también son significativos $(F=25,56, p<.000)$ a favor de las primeras. El test post-hoc también revela diferencias entre todos los pares comparados. Estos resultados permiten, por tanto, mostrar como válida la tercera de las hipótesis según la cual las cuñas radiofónicas con menor densidad informativa y velocidad de lectura obtendrían un mejor nivel de recuerdo que aquellas con índices más elevados en las dos variables. 
Por tanto, los datos indican que tan sólo en el primer bloque publicitario, el de mayor recuerdo, se ha producido tanto un efecto de primacía como de recencia, más pronunciado el primero. No en vano este bloque contenía las cuñas que reúnen más índice de recuerdo colocadas en primera y en cuarta posición. Por eso, el efecto de primacía es más sobresaliente al contener la cuña de El Corte Inglés, la de mayor recuerdo, en su posición inicial y, la segunda, La Caixa, en última posición produciendo la recencia. En cambio, se produce un descenso en los niveles intermedios más acusado en la cuña de la Fundación Repsol que aquí se encuentra en tercera posición.

En el segundo bloque más recordado, el número 3, sólo se produce un efecto de recencia pero relativo ya que se encuentra en valores cercanos a la posición segunda. Aquí las cuñas más recordadas se encuentran en la segunda posición (La Caixa) y en la última posición (El Corte Inglés), en valores muy similares. En cambio, la cuña escuchada en primera posición (Fundación Repsol) obtiene bajos niveles de recuerdo, a pesar de su colocación en primer lugar, igual que la relativa a los campeonatos, en tercera posición, si bien en menor medida que esta última.

El siguiente bloque en orden de recuerdo global ha sido el cuarto donde se produce un ligero efecto de primacía pero no de recencia. Esto se debe a que comienza con la cuña de La Caixa y obtiene el segundo lugar en recuerdo cuando se valora la primera posición de cada bloque publicitario. La segunda posición en este bloque es para la cuña de la Fundación Repsol donde se produce un importante declive del nivel de recuerdo que se vuelve a recuperar al nivel más elevado en la tercera posición para la cuña de El Corte Inglés. Por último, vuelve a descender, aunque no tanto como en segunda posición, cuando se valora la cuña última que es la de los campeonatos.

Por último, el bloque menos recordado, el número 2, vuelve a reafirmar que no se ha producido un efecto de posición serial. Aquí la curva es precisamente la contraria y es en los niveles intermedios donde se produce un aumento del recuerdo, es decir, en las cuñas de El Corte Inglés y La Caixa, con una ligera ventaja de la primera sobre la segunda. En cambio, el nivel de recuerdo es muy bajo al inicio (cuña de los campeonatos) y especialmente al final (Fundación Repsol). El gráfico 2 ilustra los resultados obtenidos: 
Fig. 2. Medias de recuerdo

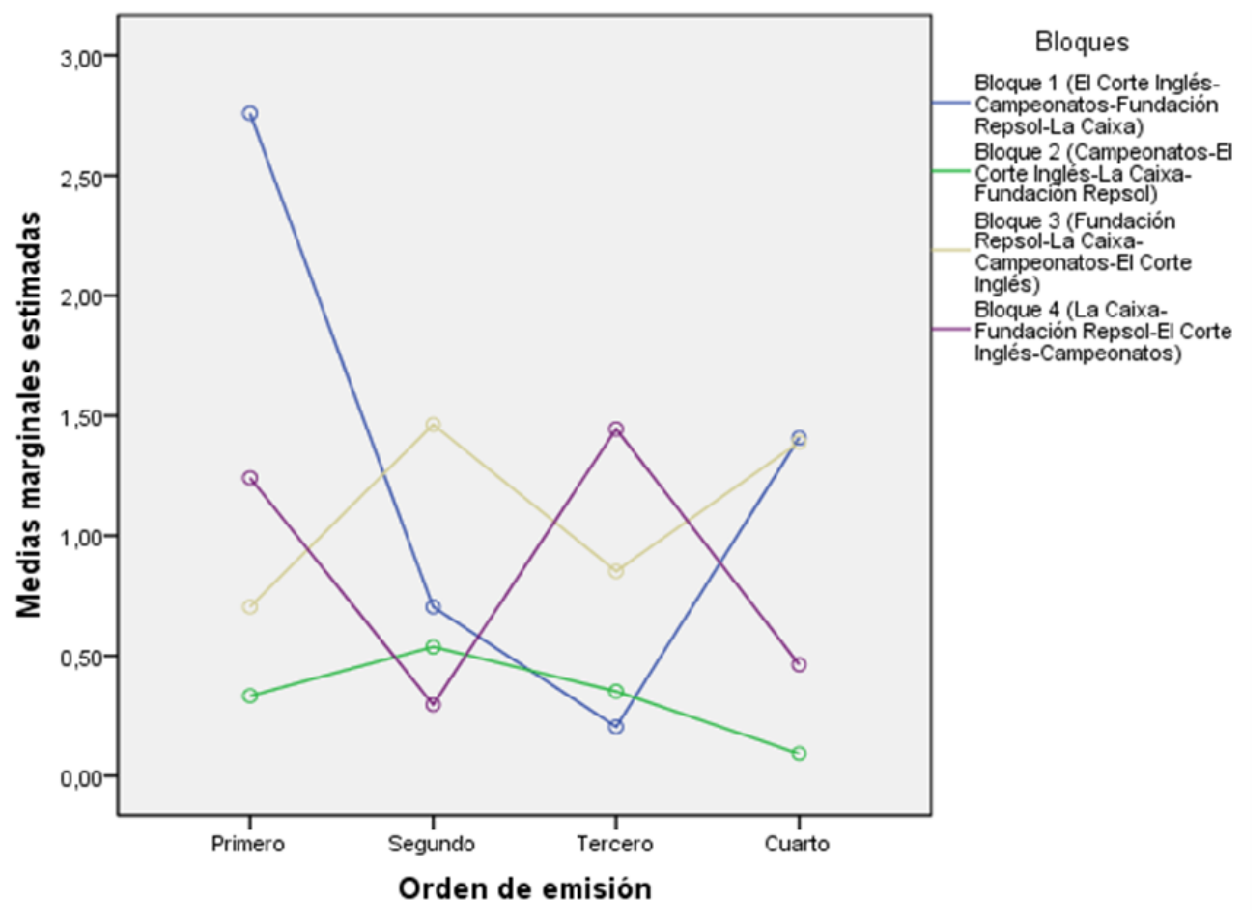

Fuente: Elaboración propia

En definitiva, los resultados indican que cuando se existe una interacción positiva entre el tipo de cuña y una posición inicial o final dentro del bloque publicitario, entonces se produce un efecto de posición serial a nivel macroestructural. En cambio, cuando el tipo de cuña presenta unas características microestructurales poco favorables, como son una alta densidad informativa y una elevada velocidad de lectura, la posición no resulta ser un factor determinante. Las características microestructurales de la cuña han sido entonces el factor que puede explicar estos resultados.

\section{Discusión}

El objetivo de este estudio era analizar el efecto de posición serial a nivel macroestructural y la posible influencia de otros factores microestructurales sobre el grado de recuerdo de un oyente expuesto a un bloque publicitario con varias cuñas radiofónicas. Son muchas las investigaciones que han constatado este efecto cuando se presenta un conjunto de estímulos, bien palabras aisladas (Glanzer y Cunitz, 1966; Murdock, 1962) o en la publicidad (Riebe y Dawes, 2006; Brunel y Nelson, 
2003; Sáiz et al., 1999; Zhao, 1997; Pieters y Bijmolt, 1997), pero siempre con resultados desiguales dependiendo de las diferentes características de la exposición al estímulo y especialmente por el estímulo en sí mismo. Así, en esta investigación, el efecto de posición serial ha sido determinante únicamente cuando las condiciones microestructurales de la cuña radiofónica han sido más favorables al recuerdo. Por tanto, ambas condiciones parecen haber influido en los resultados de este estudio, aunque en diferente grado.

En primer lugar, las características de la exposición al estímulo en forma de serie continua en un tiempo limitado con unidades autónomas sin repetición y con sujetos que partían de una baja motivación, no recibieron instrucciones previas sobre la prueba y algunos de ellos realizaban una actividad paralela constituyen un conjunto de factores macroestructurales que pueden haber contribuido a anular el efecto de posición serial. Si bien el grado de asociación entre los estímulos es un factor que nunca puede producirse por la propia naturaleza de los bloques publicitarios, la frecuencia de emisión de las cuñas sí podría haber mejorado el nivel de recuerdo. Por tanto, sería un aspecto que cabría investigar.

Pero el principal problema se produce ante la complejidad de la tarea que debían llevar a cabo los sujetos. La sensación de ruptura constante y aleatoria (Tse et al., 2004) producida por la brevedad de unas cuñas emitidas en serie parece haber limitado de manera considerable el tiempo necesario para su correcto procesamiento cognitivo. Por tanto, en esta circunstancia, los sujetos no han podido orientar los recursos necesarios para su correcta codificación y posterior recuerdo. Aquí, si se tiene en cuenta que los estudios de Potter (2009) y Tse et al. (2004) confirman un mayor nivel de recuerdo para bloques con menos anuncios, el problema no parece ser tanto el número de cuñas que lo integran como su escasa duración. Resulta sencillo deducir que si en un bloque de tan sólo cuatro cuñas los resultados son tan desfavorables al recuerdo, incrementar este número, como de hecho ocurre en la práctica radiofónica, conllevaría efectos aún más negativos. En cambio, aumentando la duración de la cuña, el oyente dispondría de más tiempo tanto para disponerse a la escucha como para procesar la información. Por eso, el estudio de Potter (2009) concluye una mayor efectividad en el recuerdo de cuñas de un minuto de duración que son emitidas en bloques. Éste sería pues un aspecto que merecería de un estudio en mayor profundidad.

Junto a ello, aunque el oyente tipo normalmente no presenta una disposición inicial a prestar atención al mensaje publicitario, un bajo grado de motivación de los sujetos hacia el estímulo podría haber limitado su capacidad de recuerdo. Bien es cierto que el hecho de que sean estudiantes de publicidad presupone un mayor interés, pero también lo es el hecho de que no son público objetivo de las cuñas del estudio. En cualquier caso, cuando los sujetos no están motivados para procesar la información, se potencia el efecto de recencia (Haugtvedt y Wegener, 1994), aspecto que en este estudio no se ha producido. Además, la cuña potencialmente más próxima a sus intereses (campeonatos) es la tercera en recuerdo, por lo que en úl- 
timo término éste no parece ser un factor determinante. No obstante, para futuras investigaciones sería de interés comprobar si los resultados difieren cuando los sujetos son potenciales destinatarios de los mensajes

Por último, tanto las condiciones de la prueba como la modalidad de escucha pueden haber incidido sobre el efecto de posición serial. La ausencia de instrucciones sobre la tarea que debían realizar después ha impedido la preparación ante el estímulo anulando el principio de primacía. Unido a ello, la realización de una actividad paralela y distractora durante la exposición serial, provocando una atención dividida hacia el estímulo, ha perjudicado notablemente la capacidad de recuerdo, como ya han comprobado algunos autores (Terry, 2005; Atkinson y Shiffrin, 1968; Postman y Phillips, 1965). En este sentido, destaca el índice tan bajo de los bloques con escucha compartida. No debemos olvidar que esta última es precisamente la condición de escucha mayoritaria entre los oyentes radiofónicos, por lo que estos bajos índices deberían hacer reflexionar seriamente sobre este aspecto.

Pero, en conjunto, a pesar de que algunos de estos factores macroestructurales pueden haber tenido su influencia sobre el efecto de posición serial y el nivel de recuerdo, no parecen ser determinantes para los resultados de este estudio. Primero, porque casi todos son inherentes a las características del mensaje publicitario radiofónico o a sus condiciones de escucha. Segundo, porque los diferentes estudios analizados muestran que, bajo estas mismas condiciones, al menos se habría de haber producido el principio de recencia, aspecto que no ocurre si no es con la interferencia de otros factores.

Por tanto y en segundo lugar, han sido las características microestructurales del mensaje publicitario, poco atractivas formalmente, con alta densidad informativa y velocidad de lectura, las que han sido determinantes en la obtención de los resultados. De hecho, el único bloque publicitario que registra el efecto de posición serial es precisamente el que contiene las cuñas más recordadas al inicio y al final.

Por sí mismo, cualquier estímulo auditivo como es el radiofónico, comporta una mayor dificultad de procesamiento y, con ello, una probabilidad más reducida de recuerdo que si estuviera completado con otros recursos (Greenfield y BeaglesRoos, 1988; Beagles-Roos y Gat, 1983). Esta puede ser la razón por la que los resultados de este estudio difieren con el efecto de posición serial que algunos autores han demostrado en mensajes publicitarios de televisión. Pero, además, si a esta dificultad previa se le añade un incremento en la complejidad del estímulo sin apoyo de elementos formales atractivos que estimulen la atención, entonces los resultados pueden ser aún más negativos, como finalmente se deduce de esta investigación. De hecho, muchos son los comentarios que los sujetos realizan sobre este aspecto: las cuñas 'son aburridas, son monótonas, no me llaman la atención, no son atractivas, son poco creativas'. Además de exponerse a un estímulo de ruptura aleatoria, bajo condiciones macroestructurales desfavorables, los sujetos han tenido que procesar un mensaje de alta complejidad, con alta densidad informativa y elevada velocidad de lectura, lo que en último término ha resultado determinante para el 
nivel de recuerdo obtenido en este estudio. En las cuñas de mayor densidad, los sujetos tenían que recordar demasiados datos (ocho y nueve respectivamente y más de tres argumentos), la mayoría de ellos cifras, expuestos en tan sólo 20 segundos. Esto implica la necesidad de incrementar los recursos asignados a la tarea y, con ello, un mayor esfuerzo cognitivo, como de hecho demuestran los comentarios de los sujetos: 'demasiada información, mucho diálogo, hay muchos datos, estoy saturado'. La densidad informativa es pues un factor a tener muy en cuenta por los publicistas porque, como bien concluye García López: «En un medio tan saturado y fugaz, con mensajes de tan corta duración, quién acaba recordando cifras, fechas, datos que se entremezclan creando una molesta confusión cuyo mejor antídoto es borrarla de la memoria»? ${ }^{7}$.

Pero al mismo tiempo que se producía la necesidad de incrementar los recursos cognitivos para codificar adecuadamente el mensaje, la exposición a una alta velocidad de lectura limitaba esta posibilidad reduciendo el tiempo necesario para el correcto procesamiento del mensaje. También en este caso han sido muchos los comentarios relativos a la velocidad del locutor: 'habla muy rápido, iba a toda velocidad, no me da tiempo a entender'. En definitiva, en último término, al no contar con suficientes recursos asignados para procesar el mensaje, se ha producido una elevada sobrecarga cognitiva (Fox et al., 2007) evidenciada en el bajo nivel de recuerdo global obtenido por las cuñas analizadas. Por tanto, este estudio de nuevo apunta a la escasa efectividad general de este tipo de publicidad radiofónica como, por otro lado, coinciden en afirmar varios autores desde hace tiempo, lo cual constituye una importante llamada de atención sobre el tipo de publicidad radiofónica que se continua haciendo en nuestro país.

\section{Referencias bibliográficas}

ASOCIACIÓN ESPAÑOLA DE LA PUBLICIDAD (2011). Informe del observatorio de la publicidad, http://www.anunciantes.com/newsletter/archivos/1102_AEA_Radio_FEBRERO_ 2011.pdf. Web visitada el 25/04/2011.

ATKINSON, R.C.; SHIFFRIN, R.M. (1968): «Human memory: A proposed system and its control processes», en Spence, K.W. y Spence, J.T. (eds.). The Psychology of Learning and Motivation: Advances in Research and Theory, New York, Academic Press, 89-195.

Ballesteros, S. (2001): Psicología general, Madrid, UNED.

Ballesteros, S.; GarcíA, B. (1998): Procesos psicológicos básicos, Madrid, Editorial Universitas.

BALSEBRE, A.; RICARTE, J.M.; PeRONA, J.J. et al. (2006): Los mitos de la publicidad radiofónica. Estrategias de la comunicación publicitaria en la radio española, Madrid, Cátedra.

Barbeito Veloso, M.; Fajula Payet, A. (2005): «La radio publicitaria: el peso del inmovilismo», Quaderns del CAC, 22, 49-62.

7 GARCÍA LóPEZ (2000), op. cit., 62. 
BeAgles-Roos, J.; GAT, I. (1983): «Specific impact of radio and television on children's story comprehension», Journal of Educational Psychology, 75, 128-137.

BoyD, A. (2003): Broadcast Journalism. Techniques of Radio and Television News, Oxford, Focal Press.

Broach, V.C.; T, Page, T.J.; WiLson, R.D. (1997): «The Effects of Program Context on Advertising Effectiveness», en Wells, W.D. (ed.), Measuring Advertising Effectiveness, Mahwah, NJ, Lawrence Erlbaum Associates.

BRUNEL, F.F.; Nelson, M.R. (2003): «Message Order Effects and Gender Differences in Advertising Persuasion», Journal of Advertising Research, 43, 330-341.

Chantler, P.; Stewart, P. (2003): Basic Radio Journalism, Oxford, Focal Press.

COHEN, J. (2001): «Defining identification: A theoretical look at the identification of audiences with media characters», Mass Communication and Society, 3, 245-264.

Corley, S.; Crocker, M.W. (2000): «The modular statistical hypothesis: Exploring lexical category ambiguity», en Crocker, M.W., Pickering, M. y Clifton, C. (eds.), Architectures and mechanisms for language processing, Cambridge, Cambridge University Press, 135-160.

FOX, J.R.; PARK, B.; LANG, A. (2007): «When Available Resources Become Negative Resources: The Effects of Cognitive Overload on Memory Sensitivity and Criterion Bias», Communication Research, 34, 277-296.

GARCÍA, F. (1999): «La publicidad en radio: imágenes de baja intensidad retórica», $L a$ publicidad en la radio. VI Jornadas de Comunicación Social, Pontevedra, Universidad de Vigo.

GARCÍA LóPEZ, M. (2000): «Creatividad radiofónica, ¿para qué?», Questiones Publicitarias, 8, 57-67.

GlanZER, M.; CUNITZ, A.R. (1966): «Two storage mechanisms in free recall», Journal of Verbal Learning and Verbal Behavior, 5, 351-360.

GoldhaBeR, G.M. (1974): «Effects of Speech Compression Training in Comprehension of Native Speakers of English and Navajo», en Duker, S. (ed.), Time Compressed Speech: An Anthology and Bibliography, Metuchen, NJ, The Scarecrow Press, Inc, 730-735.

Greenfield, P.M.; Beagles-Ross, J. (1988): «Radio vs. television: Their cognitive impact on different socio-economic groups», Journal of Communication, 38, 71-92

Haugtvedt, C.; Wegener, D.T. (1994): «Message Order Effects in Persuasion: An Attitude Strength Perspective», Journal of Consumer Research, 21(1), 205-18.

HunT, R.R. (1995): «The subtlety of distinctiveness: what von Restorff really did», Psychonomic Bulletin and Review, 2, 105-112.

INFOADEX (2011): Estudio de la inversión publicitaria en España, febrero de 2011.

JAHNKE, J.C. (1968): «Presentation Rate and the Serial-Position Effect of Immediate Serial Recall», Journal of Verbal Learning and Verbal Behavior, 7, 608-612.

JOANNIS, H. (1996): La creación publicitaria desde la estrategia de marketing, Bilbao, Deusto.

JURAFSKY, D.A. (1996): «Probabilistic model of lexical and syntactic access and disambiguation», Cognitive Science, 20, 137-194.

LANA, R.E. (1963): «Interest, Media, and Order Effects in Persuasive Communications», Journal of Psychology, 56(1), 9-13. 
LANG, A. (2009): «The limited capacity model of motivated mediated message processing», en Nabi, R. y Oliver, M.B. (eds.). The SAGE Handbook of Mass Media Effects, USA, Sage publication, 193-204.

- (2006): «Using the Limited Capacity Model of Motivated Mediated Message Processing to Design Effective Cancer Communication Messages», Journal of Communication, 56 (s1), S57-S80.

- (2000): «The limited capacity model of mediated message processing», Journal of Communication, 50(1), 46-70.

LI, C. (2010): «Primacy effect or recency effect? A long-term memory test of Super Bowl commercials», Journal of Consumer Behavior, 9, 32-44.

MARCOS, I. (2006): «Creatividad: viejas historias de la radio», Anuncios, 30, 20-21.

Meyerson, M.D. (1974): «An Exploration of Comprehension Differences in TimeCompressed Japanese, Chinese, Indi and English Speech», en Duker, S. (ed.), Time Compressed Speech: An Anthology and Bibliography, Metuchen, NJ, The Scarecrow Press, Inc, 730-735.

Muela Molina, C. (2008): «La representación de la realidad en la cuña radiofónica», Comunicación y Sociedad, 21(2), 115-139

- (2007): «El surrealismo en la cuña radiofónica. La dimensión de los absurdo al servicio de la comunicación comercial», Espéculo: Revista de Estudios Literarios, 37.

- (2001): La publicidad radiofónica en España. Análisis creativo de sus mensajes, Madrid: Ediciones Internacionales Universitarias.

Murdock, B.B. Jr. (1962): «The Serial Position Effect of Free Recall», Journal of Experimental Psychology, 64(5), 482-488.

MurPheY, C.; DoBIE, K.; Grant, J. (2003): «Time versus Pause Manipulation in Communications Directed to the Young Adult Population: Does It Matter?», Journal of Advertising Research, 43, 281-292.

OBERAUER, K. (2003): «Understanding serial position curves in short-term recognition and recall», Journal of Memory and Language, 49(4), 469-483.

PÉrez, M. (2006): «Tony Hertz, creativo de Hertz Radio», El Publicista, 138, 50-51.

PERONA PÁEZ, J.J. (2007): «Formatos y estilos publicitarios en el prime-time radiofónico español: infrautilización y sequía de ideas», Zer, 23, 219-242.

Perona PÁez, J.J.; BArbeito Veloso, M.L. (2008): «El lenguaje radiofónico en la publicidad del prime time generalista. Los anuncios en la 'radio de las estrellas'», Telos, 77, 115-124.

PIETERS, R.G.M.; BiJMOLT, T.H.A. (1997): «Consumer memory for television advertising: a field study of duration, serial position, and competition effects», Journal of Consumer Research, 23(4), 362-372.

Postman, L.; PhiLliPS, L.W. (1965): «Short term temporal changes in free recall», Quarterly Journal of Experimental Psychology, 17, 132-138.

POTTER, R.F. (2009): «Double the Units: How Increasing the Number of Advertisements while Keeping the Overall Duration of Commercial Breaks Constant Affects Radio Listeners», Journal of Broadcasting \& Electronic Media, 53(2), 584-598.

PotTer, R.F.; WANG, Z., ANGELini, J.R. et al. (2006): «The effect of structural complexity and information density on cognitive effort and arousal during audio message processing», Psychophysiology, 43, S79-S79. 
PotTer, R.F.; CHOI, J. (2006): «The Effects of Auditory Structural Complexity on Attitudes, Attention, Arousal, and Memory», Media Psychology, 8, 395-419.

PotTER, R.F.; CALlisOn, C. (2000): «Sounds exciting!! The effects of auditory complexity on listeners' attitudes and memory for radio promotional announcements», Journal of Radio Studies, 7, 29-51.

PRESS, A. (1989): «Class and gender in the hegemonic process: Class differences in women's perceptions of television realism and identification with television characters», Media, Culture and Society, 11, 229-251.

RiEBE, E.; DAwES, J.G. (2006): «Recall of Radio Advertising in Low and High Clutter Formats», International Journal of Advertising, 25(1), 71-86.

Rodero ANTÓn, E. (2008): «Publicidad en radio sí, pero no radiofónica», Área Abierta, 20, $1-16$.

- (2007): «Caracterización de una correcta locución informativa en los mensajes audiovisuales», Estudios del mensaje periodístico, 13, 523-542.

Rodero, E.; LARREA, O.; VÁZQUEZ, M. (2010): «Voces masculinas y femeninas en la locución de cuñas radiofónicas. Estudio sobre la efectividad y su adecuación con el producto», Icono 14, A4, 281-295.

Rodero Antón, E.; Alonso GonzÁlez, C.M.; Fuentes ABAD, J.A. (2004): La radio que convence. Manual para creativos y locutores publicitarios, Barcelona, Ariel.

RUNDUS, D. (1971): «Analysis of research processes in free recall», Journal of Experimental Psychology, 89, 63-77.

SÁIZ, M.; BAQUÉS, J.; SÁIZ, D. (1999): «Factores que pueden mejorar la codificación de los mensajes publicitarios: ¿una cuestión de memoria implícita o de memoria explícita?», Psicothema, 11(4), 891-900.

TANNENBAuM, P.H. (1954): «Effects of Serial Position on Recall of Radio News Stories», Journalism Quarterly, 31(3), 318-323.

TERRY, S.W. (2005): «Serial position effects in recall of television commercials», Journal of General Psychology, 132(2), 151-164.

TSE, P. U.; InTRILIGATOR, J.; RiVEST, J. et al. (2004): «Attention and the subjective expansion of time. Perception and Psychophysics», 66(7), 1171-1189.

VÁZQUEZ GESTAL, M. (2001): «La infravaloración publicitaria del medio radio», Revista Latina de Comunicación Social, 37.

WEBB, P.H.; RAY, M.L. (1979): «Effects of TV clutter», Journal of Advertising Research, 19(3), 7-12.

ZHAO, X. (1997): «Clutter and serial order redefined and retested», Journal of Advertising Research, 37(5), 57-73. 
Anexo. Cuestionario del estudio

\section{CUÑA 1. El Corte Inglés}

1. ¿De qué te intentará convencer un amigo?

2. ¿Qué intentará hacer tu amigo?

3. ¿Qué característica tienen los productos que se anuncian?

4. ¿Dónde se venden?

5. ¿Qué marca se anuncia?

6. ¿Qué producto concreto se anuncia?

CUÑA 2. Campeonatos de Trial y Endur Indoor

1. ¿Cómo se califica el cóctel que hacen?

2.¿Qué quieren ser los pilotos catalanes?

3. ¿Cuáles son los dos campeonatos del mundo que se celebran?

4. ¿Dónde se celebren les pruebas?

5. ¿En qué fecha de la semana se celebran?

6. ¿Dónde se venden las entradas?

CUÑA 3. Fundación Repsol

1. ¿Para qué sirve ahorrar energía?

2. ¿Mediante qué acción que se celebrará puedes conocer la manera de ahorrar energía?

3. ¿Con qué actividades se desarrollará la acción?

4. ¿En qué dos lugares se desarrollará la acción?

5. ¿En qué fecha?

6. ¿Qué marca se anuncia?

\section{CUÑA 4. La Caixa}

1. ¿De qué aspectos se ocupa esta empresa que te deben interesar, según el anunciante?

2. ¿Qué regalo puedes ganar?

3. ¿Cuál es la condición para ganar el regalo?

4. ¿De qué cantidad económica es el regalo?

5. ¿Dónde te puedes informar?

6. ¿Qué marca se anuncia? 